\title{
FOLGT AUS DEM UNWERT DER TIERHALTUNG EIN VERBOT DES FLEISCHKONSUMS?*
}

\author{
Simon Gaus
}

It is natural to assume that it can only be morally permissible for consumers to buy meat products if the breeding and killing of animals for the purpose of meat production is morally acceptable. This assumption presupposes a stable and morally relevant connection between the consumption and the production of meat. While both actconsequentialism and the Kantian idea of generalizability initially appear to support that view, neither of them succeeds in establishing a connection of the required kind.

Viele, die eine vegetarische Lebensweise für moralisch geboten halten, gehen von folgender Annahme aus: Wenn die Praxis der Fleischproduktion - die Zucht, Haltung und Schlachtung von Tieren - unter Berücksichtigung aller positiven und negativen Folgen für Menschen, Tiere und Umwelt moralisch inakzeptabel ist, dann ist es moralisch geboten, auf den Konsum von Fleisch zu verzichten. ${ }^{1}$ Wer diese Ansicht vertritt, ist der Auffassung, dass zwischen Fleischkonsum und Fleischproduktion ein moralisch bedeutsamer Zusammenhang besteht.

Ich diskutiere im Folgenden zwei naheliegende Kandidaten für einen solchen Zusammenhang. Der erste Kandidat ist die handlungskonsequentialistisch motivierte Idee, dass der moralische Wert der einzelnen Konsumhandlungen vom moralischen Wert der Fleischproduktion abhängt, weil die einzelne Konsumhandlung zur Fortführung der Fleischproduktion beiträgt. Der zweite Ansatz geht aus von der Annahme, dass kein Fleisch produziert würde, wenn alle auf den Fleischkonsum verzichten würden und dass Fleischkonsum daher einer Verallgemeinerbarkeitsbedingung nicht gerecht wird, sofern die Praxis der Fleischproduktion moralisch schlecht ist. Es wird sich zeigen, dass keiner der beiden Ansätze es erlaubt, aus der (angenommenen) Schlechtigkeit der Fleischproduktion eine stabile moralische Pflicht, auf den Fleischkonsum zu verzichten, abzuleiten. Die Verallgemeinerbarkeitsbedingung legt vielmehr nahe, dass, wenn die Fleischproduktionspraxis insgesamt schlecht ist, eine vegetarische Lebensweise supererogatorisch ist.

\footnotetext{
* Für hilfreiche Kommentare und Hinweise danke ich Cécile Bonneton, Anne Burkard, Christoph Fehige, Jan Gertken, Romy Jaster, Kirsten Meyer, Andreas Müller, Daniel Ramöller und Thomas Schmidt, sowie zwei anonymen Juroren des Essaypreises der Gesellschaft für Analytische Philosophie und der Grazer Philosophischen Studien.

${ }^{1}$ So etwa in (Singer, 1976). Allgemein scheint jede Begründung des moralischen Vegetarismus, die den Wert tierischen Lebens und Leidens in den Vordergrund rückt, die genannte These vorauszusetzen.
} 


\section{Handlungskonsequentialismus}

Der Ausgangspunkt handlungskonsequentialistischen Nachdenkens ist die Vorstellung, dass wir alle darauf abzielen sollten, die Welt zu verbessern - soweit uns das eben möglich ist. Wir können diese Idee klarer fassen als

Konsequentialismus-Prinzip: Wenn für zwei Handlungsoptionen A und B gilt, dass die Welt insgesamt besser werden würde, wenn A, als wenn B ausgeführt würde, dann soll A ausgeführt werden. ${ }^{2}$

Das Konsequentialismus-Prinzip kann nur dann aus der Schlechtigkeit der Fleischproduktion ein Konsumverbot generieren, wenn die einzelnen Konsumentscheidungen tatsächlich einen Einfluss darauf haben, wie und wie viel produziert wird. Damit das überhaupt plausibel ist, wird „Konsum“ hier wohl als Kauf und nicht als Verzehr zu verstehen sein - denn ob das Fleisch, nachdem es gekauft wurde, verzehrt wird oder nicht, wird vom Produzenten nicht registriert. Die naheliegende Vermutung ist, dass die Menge des produzierten Fleisches von der Gesamtnachfrage nach Fleisch abhängig ist, dass jeder Kaufakt zur Gesamtnachfrage beiträgt, und dass deshalb für jeden Kaufakt gilt, dass etwas weniger Fleisch produziert worden wäre, wenn er nicht getätigt worden wäre.

Der letzte Schritt in dieser Überlegung scheint allerdings nicht zu stimmen. Denn obwohl Produzenten ihre Produktion natürlich an Veränderungen in der Gesamtnachfrage anpassen, werden minimale Nachfrageschwankungen in der Regel keinerlei Veränderung in der Produktion bewirken. Endproduzenten registrieren die Entscheidung eines einzelnen Käufers, ein Huhn zu kaufen oder nicht zu kaufen, oft gar nicht. Es ist deshalb für jede einzelne Kaufentscheidung sehr unwahrscheinlich, dass das Unterlassen dieses Kaufes irgendetwas am Verhalten der Endproduzenten geändert hätte. ${ }^{3}$ In aller Regel machen unsere einzelnen Kaufentscheidungen also keinen Unterschied für das Leben oder Wohlergehen auch nur eines einzelnen Tieres. ${ }^{4}$ Daraus scheint zu folgen, dass der Handlungskonsequentialismus aus der Schlechtigkeit der Produktionspraxis kein allgemeines Gebot, auf Fleischkonsum zu verzichten, generieren kann.

\footnotetext{
${ }^{2}$ Ich folge in der Formulierung (Kagan, 2011), (Schwartz, 2010), (Norcross, 2004), (Glover, 1975) und anderen.

${ }^{3}$ Diese Annahme ist unter Verteidigern des konsequentialistischen Ansatzes verhältnismäßig unkontrovers; vgl. (Kagan, 2011) und (Singer, 1980, S. 336).

${ }^{4}$ Man mag hier auf externe Faktoren hinweisen, insbesondere auf den Einfluss des eigenen Verhaltens auf andere Konsumenten durch Vorbildfunktionen (vgl. (Glover, 1975)). Diese Strategien haben aber zwei Probleme: Erstens ist das, wozu wir dann verpflichtet sind, nur noch mittelbar mit dem Kauf oder Verzehr von Fleisch verbunden; zweitens ist auch bei unserer Wirkung auf andere nicht klar, dass der einzelne beispielhafte Kaufakt einen Unterschied für hinreichend viele Kaufentscheidungen anderer Personen macht.
} 
Shelly Kagan (Kagan, 2011) hat diese Schlussfolgerung kürzlich zurückgewiesen. ${ }^{5}$ Kagan geht von der Annahme aus, dass eine Handlungsoption genau dann geboten ist, wenn der $z u$ erwartende Nutzen dieser Option höher ist als der $\mathrm{zu}$ erwartende Nutzen aller anderen Handlungsoptionen. Der zu erwartende Nutzen einer Handlungsoption wird ermittelt, indem man jedes mögliche Resultat dieser Option mit der subjektiven Eintrittswahrscheinlichkeit dieses Resultats (unter der Bedingung, dass die betrachtete Handlungsoption ausgeführt wird) multipliziert und die Summe all dieser Produkte bildet. ${ }^{6}$

Angewandt auf den einzelnen Fleischkauf: Der positive Teil des Nutzens eines Fleischkaufs besteht im resultierenden Fleischgenuss. Das negative Resultat besteht in der Möglichkeit, dass der jeweilige Akt des Fleischkonsums einen Unterschied für den Umfang der Fleischproduktion, und damit für das Wohlergehen von Tieren, macht. Umso gewichtiger der potentielle Unterschied (umso größer beispielsweise die Zahl der potentiell betroffenen Tiere), und umso höher die Wahrscheinlichkeit, dass der betrachtete Kaufakt tatsächlich diese Wirkung hat - der jeweilige Kaufakt etwa zur Tötung zusätzlicher Tieres führt -, desto kleiner ist der zu erwartende Nutzen des Fleischkaufs im Vergleich zur Verzichtsoption.

Nehmen wir an, dass die aggregierte Menge der Lust, die durch den Fleischgenuss aller Konsumenten entsteht, die aggregierten tierischen Leiden und anderen negativen Folgen der gesamten Fleischproduktion nicht aufwiegt. Wenn sich nun die Verursachung des aggregierten Leids (sowie die Genusslust) einigermaßen gleichmäßig auf alle einzelnen Fleischkäufe verteilte, dann hätte jeder einzelne Fleischkauf einen geringeren zu erwartenden Nutzen als die Verzichtsoption. Dieser Fall ist der perfekter Nachfragesensitivität - der Produzent reagiert auf jeden einzelnen Kauf mit der Neuproduktion genau der Menge, die gekauft wurde.

Von dieser Idealisierung ausgehend versucht Kagan zu zeigen, dass der zu erwartende Nutzen einer Kaufhandlung von Veränderungen der Nachfragesensitivität des Produzenten gänzlich unberührt bleibt. Kagans Grundidee ist diese: Zwar sinkt bei abnehmender Nachfragesensitivität die Wahrscheinlichkeit, dass eine einzelne Kaufentscheidung einen Unterschied für die produzierte Menge macht. Gleichzeitig steigt die Größe des Unterschiedes, den die einzelne Kaufhandlung macht, wenn der Fall des Unterschiedmachens eintritt, jedoch an - und zwar im selben Maße, in dem die Eintrittswahrscheinlichkeit abnimmt. Der zu erwartende Nutzen einer Kaufhandlung - das

\footnotetext{
${ }^{5}$ Tatsächlich ist Kagans Argumentation sehr nah an Peter Singers Reaktion (Singer, 1980, S. 335) auf einen fast identischen Einwand in (Wentz, 1979), (Devine, 1978), (Regan, 1980) und anderen.

${ }^{6}$ Das entspricht der Verwendung von „expected utility“ in (Kagan, 2011).
} 
Produkt aus dem Nutzen des Effektes und der Wahrscheinlichkeit seines Eintretens - bliebe so immer unverändert. Im folgenden Stelle ich Kagans Argumentation im Detail vor.

Klarerweise, so Kagan, reagieren Produzenten auf starke Veränderungen der Gesamtnachfrage durch Erhöhung oder Verringerung der Produktion. Wenn also nicht auf jeden einzelnen Kauf reagiert wird, dann muss das daran liegen, dass der Produzent in gröberen Abstufungen reagiert - etwa nur die Produktion anpasst, wenn eine hinreichend große Menge zusätzlicher Produkte verkauft wurde. Damit allerdings nicht dauerhaft zu viel oder zu wenig produziert wird, muss die Menge, die beim Erreichen einer neuen Stufe zusätzlich produziert wird, dem Abstand zwischen den Reaktionsstufen entsprechen. Wenn beispielsweise die Produktion angepasst wird, sobald zehn Hühner mehr nachgefragt werden, muss die Anpassung in der „Produktion“ von jeweils zehn Hühnern mehr bestehen.

Der einzelne Kauf macht in diesem Bild genau dann einen Unterschied, wenn eine Stufe im zugehörigen Zeitabschnitt (Tag, Woche etc.) genau erreicht wurde - denn dann, und nur dann, gilt für jeden einzelnen Kauf, dass, wenn er nicht getätigt worden wäre, weniger Tierfleisch produziert worden wäre. Liegen also, wie in unserem Beispiel, die Reaktionsstufen bei jeweils zehn zusätzlichen Käufen, so macht genau dann jeder einzelne Kauf einen Unterschied, wenn die Verkaufsmenge ein Vielfaches von zehn ist.

Da die einzelne Kaufhandlung genau dann einen Unterschied für die Produktion macht, wenn die Verkaufsmenge ein Vielfaches des Abstands zwischen den Reaktionsstufen ist, entspricht die subjektive Wahrscheinlichkeit dafür, dass die einzelne Kaufhandlung einen Unterschied macht, der subjektiven Wahrscheinlichkeit dafür, dass die Verkaufsmenge ein Vielfaches des Stufenabstandes ist.

Der Clou in Kagans Argument ist nun dieser: Die subjektive Wahrscheinlichkeit aller möglichen Verkaufsmengen, so Kagan, ist gleich - dass genau 10 Hühner verkauft werden, ist subjektiv ebenso wahrscheinlich, wie dass 11, 12, 13 etc. Hühner verkauft werden. Wenn der Abstand zwischen den Reaktionsstufen bei 10 Hühnern läge, dann wäre die Wahrscheinlichkeit dafür, dass die Verkaufsmenge ein Vielfaches des Stufenabstands beträgt, genau 1/10 - weil jede zehnte mögliche Menge ein Vielfaches von 10 ist. Lägen die Stufen bei jeweils zwanzig zusätzlichen Hühnern, wäre die Wahrscheinlichkeit 1/20, und so fort. Die Wahrscheinlichkeit dafür, dass der einzelne Kauf einen Unterschied macht, ist also 1/(Stufenabstand).

Die Menge, die beim Erreichen einer Stufe zusätzlich produziert werden würde, entspricht aber immer genau dem Stufenabstand - das ergab sich oben aus der Notwendigkeit, Überoder Unterproduktion zu verhindern. Wenn also auf jeweils zehn zusätzliche verkaufte 
Hühner reagiert wird, dann besteht für jeden Kauf jeden Tag eine 1/10 Chance, den Tod von 10 Hühnern zu verursachen. Beträgt der Stufenabstand 1 (perfekte Sensitivität), hat jeder Kauf eine Chance von 1, den Tod von einem Huhn zu verursachen. Reagiert der Produzent nur in Tausenderstufen, gibt es eine Chance von 1/1000, den Tod von eintausend Hühnern zu verursachen. Der Erwartungsnutzen der Kaufhandlung ist in all diesen Fällen gleich jedenfalls, sofern sie sich lediglich hinsichtlich der Nachfragesensitivität des Produzenten unterscheiden. Wenn das stimmt, dann gibt es aus konsequentialistischer Sicht keinen moralisch relevanten Unterschied zwischen relativ nachfrage-insensitiv und perfekt sensitiv agierenden Produzenten.

Soweit Kagans Argument. Hier ist mein Einwand: Es stimmt nicht, dass die subjektive Wahrscheinlichkeit, eine Reaktionsstufe genau zu erreichen, genauso hoch ist wie die subjektive Wahrscheinlichkeit jeder anderen Verkaufsmenge. Nehmen wir an, ein Produzent verkauft in einer normalen Woche zwischen 5600 und 5900 Hühner und schlachtet wöchentlich 6000 Hühner. Nehmen wir weiter an, die Stufen, ab der der Anbieter auf Nachfrageschwankungen reagiert, haben einen Abstand von 500 - der Produzent reagiert also mit Produktionsanpassungen, sobald 500, 1000, 1500, mehr Hühner als erwartet verkauft wurden. Die Wahrscheinlichkeit dafür, dass eine dieser Stufe genau erreicht wird, entspricht in diesem Fall nicht einfach dem Anteil der Vielfachen von 500 an der Menge der möglichen Verkaufsmengen. Denn in dem Bereich der normalen Verkaufsmengen liegt kein einziges Vielfaches von 500, und die Eintrittswahrscheinlichkeit jeder normalen Verkaufsmenge ist höher als die jeder außerhalb des Normalbereichs liegenden.

Fälle dieser Art treten ein, wenn es einen stabilen Bereich normaler Verkaufsmengen gibt und die erste Stufe, ab der der Produzent auf Nachfrageschwankungen reagiert, außerhalb dieses Normalbereichs liegt. Umso stabiler die Nachfrage ist, desto eher wird auch die erste Reaktionsstufe außerhalb des Normalbereiches liegen. Das liegt zum einen einfach daran, dass die Wahrscheinlichkeit, dass die erste Stufe in dem Normalbereich liegt, umso kleiner wird, je „enger“ der Normalbereich ist. Hinzu kommt aber, dass Nachfragesensitivität bei hinreichend stabiler (und so auch prognostizierbarer) Nachfrage vor allem die Funktion haben wird, auf unerwartete, extreme Schwankungen reagieren zu können.

Wenn diese Beschreibung auf die Fleischproduktion zutrifft, dann nimmt - im Gegensatz zu Kagans Darstellung - bei zunehmender Nachfrageinsensitivität die Wahrscheinlichkeit, dass die einzelne Konsumentscheidung eine Wirkung auf die Produktionsmenge macht, schneller ab, als die Größe der potentiellen Wirkung zunimmt. Der zu erwartende Nutzen des einzelnen 
Fleischkaufs nimmt (auch im Vergleich zur Verzichtsoption) in solchen Fällen mit sinkender Nachfragesensitivität ab.

Das bedeutet: Wenn der Konsument gern Fleisch isst, die Gesamtnachfrage hinreichend stabil und die Nachfragesensitivität des Produzenten hinreichend grob ist, dann ist der zu erwartende Nutzen des Kaufens von Fleisch selbst dann positiv, wenn es (wie oben vorausgesetzt) insgesamt besser (oder nützlicher) wäre, wenn kein Fleisch produziert würde und kein Mensch Fleisch genießen könnte. ${ }^{7}$

Dass es von (bestenfalls) offenen empirischen Fragen abhängt, ob das KonsequentialismusPrinzip ein Konsumverbot etabliert, macht es als Bezugspunkt für die Begründung eines stabilen Zusammenhangs zwischen der Schlechtigkeit der Fleischproduktion und dem Verbot von Fleischkonsum einigermaßen unattraktiv. Hinzu kommt jedoch ein weiteres Problem: Wenn wir die Wahl haben, entweder von relativ kleinen, nachfrage-sensitiv produzierenden Anbietern zu kaufen, oder von großen, insensitiv produzierenden, so könnte uns die angeführte konsequentialistische Überlegung einen Grund geben, Fleisch vom verschwenderischen Massentierhalter zu kaufen - und dieser Grund würde gar aus dem Unwert tierischen Leidens folgen.

Man könnte vermuten, dass dies nur deshalb absurd erscheint, weil wir insgeheim unterstellen, dass die Tiere in der Massentierhaltung wesentlich mehr leiden als die Tiere, die von kleineren Betrieben gehalten werden. Doch selbst wenn Tiere in Massentierhaltung wesentlich mehr leiden als in kleinen Betrieben (aber die Tiere auch in den kleinen Betrieben so viel leiden, dass es alles in allem besser wäre, wenn die Tiere gar nicht gehalten würden), kann uns der Unwert tierischen Leidens einen entscheidenden Grund geben, Fleisch aus Massentierhaltung zu kaufen - nämlich dann, wenn der Unterschied in der Nachfragesensitivität zwischen Klein- und Großproduzent hinreichend groß ist.

Wer also glaubt, dass aus dem moralischen Wert von tierischem Wohlergehen nicht ein Grund folgen kann, Fleisch aus industrieller Massentierhaltung statt aus kleinerer Produktion zu kaufen - und zwar einfach dadurch, dass die Nachfrage stabil genug ist und der Produzent gelegentliche Überproduktion in Kauf nimmt -, der sollte sich zur Begründung der Vegetarismus-Pflicht nicht auf die dargestellten handlungskonsequentialistischen Überlegungen berufen.

\footnotetext{
${ }^{7}$ Ich setze voraus, dass die Chance, für das Leben von Tieren einen Unterschied zu machen, mit dem persönlichen ,Schaden' des Nicht-Genusses von Fleisch verrechnet wird - das also die angesetzte Werttheorie durch Fleischgenuss entstehender Lust positiven Wert zuschreibt. Man könnte demgegenüber behaupten, dass jede Handlung, die die Chancen, dass Tiere leiden, erhöht, eben dadurch verboten ist - ganz egal, wie gut das Ausführen der Handlung für die eigene Person wäre. Eine solche These ist jedoch gerade für den Bereich kleiner Wahrscheinlichkeiten problematisch: Für fast jede unserer Handlungen besteht ein minimales Risiko, dass sie durch seltsame Kausalketten zur Schädigung anderer Menschen führt.
} 


\section{Verallgemeinerbarkeit}

Es liegt hier sehr nahe, mit dem Hinweis $\mathrm{zu}$ reagieren, dass zwar einzelne Kaufentscheidungen keinen Unterschied machen mögen, dass aber der Fleischverzicht hinreichend vieler Menschen zur Verringerung der Produktionspraxis führen würde. Dieser Gedanke legt nahe, dass sich der Fleischkonsum auf der Basis einer VerallgemeinerbarkeitsBedingung als moralisch falsch erweisen lässt. Wenn jeder bereit wäre, Fleisch zu kaufen, wäre die Welt deutlich schlechter, als wenn jeder auf Fleisch verzichten würde - deshalb, so könnte das Argument lauten, sollte jeder auf Fleisch verzichten. ${ }^{8}$

So einfach ist die Sache jedoch nicht. Die kollektive Bereitschaft, Fleisch zu konsumieren, ist allein nicht hinreichend für das Leiden von Tieren - es bedarf zusätzlich des Schlachtens, d.i. der Bereitschaft einiger Menschen, aktiv Tiere zu züchten und zum Zwecke der Fleischproduktion zu töten. Das hat die Konsequenz, dass die Folgen der universellen Befolgung der Regel „Verzichte auf das Kaufen von Fleisch“ - also das, was passieren würde, wenn jeder so handelte, wie in der Regel verlangt -, keinen Deut besser sind als die Folgen der universellen Befolgung der Regel „Töte keine Tiere und iss, worauf du Lust hast“. Der Kauf von Fleisch ist zwar von der erstgenannten Regel verboten, von der letztgenannten jedoch nicht - denn der einzelne Kaufakt ist kein Tötungsakt. Da beide Regeln hinsichtlich der Folgen ihrer universellen Befolgung gleich gut sind, gibt es eine hinsichtlich ihrer Universalisierbarkeit ideale Regel, die das Kaufen von Tierfleisch nicht verbietet. Das sollte ausreichen, damit die Handlung des Fleisch-Kaufens als universalisierbar zählt. ${ }^{9}$ Die Forderung nach Universalisierbarkeit kann deshalb kein Verbot des Fleischkonsums generieren.

Man könnte hier entgegnen, dass die Befolgung der erstgenannten Regel (Konsumverbot) selbst dann ideale Konsequenzen hat, wenn weiterhin manche Leute zu Regelverletzungen bereit sind, was im Falle der letztgenannten Regel (Nicht-Tötung) dagegen nicht gilt. Diese Erwiderung lässt sich auf zwei Weisen ausbuchstabieren. Einmal kann man auf die Realität hinweisen - de facto sind manche Leute bereit, Tiere zu schlachten, und die Frage ist nun, wie wir damit umgehen. So zu denken liegt zwar nahe, ist aber im Kontext einer Universalisierbarkeitsforderung letztlich nicht sinnvoll. Denn die Idee der Verallgemeinerbarkeit basiert ja gerade darauf, dass von dem tatsächlichen Verhalten der

\footnotetext{
8 Die Forderung der Verallgemeinerbarkeit spielt eine zentrale Rolle u.a. in regelkonsequentialistischen Moraltheorien sowie in M.G. Singers Argument der Verallgemeinerung (Singer, 1961).

${ }^{9}$ Ich setze hier voraus, dass eine Handlung nur dann nicht-universalisierbar ist, wenn alle wohlgeformten Regeln die universalisierbar sind, die Handlung verbieten. Andernfalls wäre die Universalisierbarkeitsforderung viel zu stark: Für viele erlaubte, aber nicht gebotene Handlungen wird es einige universalisierbare Regeln geben, die mit dem Ausführen der erlaubten Handlung nicht kompatibel sind. Das sollte nicht dazu führen, dass diese Handlungen am Universalisierbarkeitskriterium scheitern.
} 
Menschen abstrahiert wird - ausgerechnet bei der Tötungsbereitschaft die Abstraktion zu beenden, erscheint völlig unmotiviert. ${ }^{10}$

Zweitens, und vielversprechender, kann man darauf verweisen, dass die Konsum-Regel bei breiter, aber nicht-universeller Befolgung bessere Konsequenzen hätte als die Nicht-TötungsRegel, und behaupten, dass diejenige von zwei universalisierbaren Regeln zu befolgen ist, deren Konsequenzen bei nicht-universeller Befolgung besser sind. ${ }^{11}$ Es ist leicht zu sehen, dass sich so ein Konsumverbot begründen ließe. Nur ist das hier vorgeschlagene Kriterium auch erheblich stärker als typische, durch die Ideen moralischer Unparteilichkeit und interpersoneller Fairness motivierte und begründete Verallgemeinerbarkeitsforderungen.

Besonders deutlich wird das am Beispiel Weltarmut: Angenommen, die Welt wäre insgesamt am besten, wenn jeder Bürger der reicheren Staaten mindestens 10\% seines Einkommens spenden würde - die resultierende Verteilung wäre dann ideal. Wenn gefordert ist, eine Regel zu befolgen, die bei universeller Befolgung beste Konsequenzen hätte, so wäre jeder lediglich verpflichtet, $10 \%$ seines Einkommens zu spenden. Wäre es nun aber gefordert, eine Regel zu befolgen, deren nicht-universelle Befolgung schon beste Konsequenzen hätte, dann wäre es von jedem gefordert, mehr als seinen Anteil - 10\% des Gehalts - zu spenden. Hier wird deutlich: Das Prinzip, diejenige Regel zu befolgen, deren nicht-universelle Befolgung schon beste Konsequenzen hätte, ist deutlich stärker als die Forderung nach Unparteilichkeit und gleicher Lastenverteilung, die oft im Hintergrund des Universalisierbarkeitsgedankens steht. Denn ersteres impliziert im Gegensatz zum letzteren Gedanken die Pflicht, für das regelverletzende Handeln anderer zu kompensieren. Sicherlich unterliegen wir in vielen Fällen solchen Pflichten, aber ihre Begründung scheint nicht mehr viel mit der Forderung zu tun zu haben, zu bedenken, was geschehen würde, wenn alle dasselbe täten.

Man könnte hier einwenden, dass der Verzicht auf Fleisch keine Kompensationsleistung für das Fehlverhalten der Schlachter im gleichen Sinne ist, weil ohnehin kein Fleisch zur Verfügung stände, wenn alle die Nicht-Tötungs-Regel befolgten. Die Vegetarierin, so der Einwand, gibt also im Gegensatz zur kompensierenden Spenderin nichts von dem auf, was sie hätte, wenn die Welt moralisch ideal wäre.

Hierauf lässt sich auf zwei Weisen reagieren. Zum einen habe ich lediglich zeigen wollen, dass das Prinzip, das wir anlegen müssen, um zu zeigen, dass die „Verzichte auf Fleisch“Regel besser als die „Töte keine Tiere“-Regel ist, Kompensationspflichten impliziert und

\footnotetext{
${ }^{10}$ Eine strukturell analoge Überlegung findet sich bei M.G. Singer (Singer, 1961, Ch. 3, §7).

${ }^{11}$ Brad Hookers Regelkonsequentialismus (Hooker, 2000) scheint ein Beispiel hierfür zu sein, insofern hier nicht die Folgen nicht einer universellen, sondern einer 90\%igen Befolgung der jeweiligen Regel für die moralische Beurteilung relevant sind.
} 
damit substantiell über ein Universalisierbarkeitskriterium hinausgeht. Ob der Verzicht auf Fleisch selbst eine Kompensationsleistung ist, ist dafür zunächst unerheblich.

Zum anderen scheint es mir aber durchaus einen Unterschied zu geben zwischen dem bewussten Verzicht auf und der Unverfügbarkeit von Fleisch. Im einen Fall muss einer Versuchung widerstanden werden, im anderen nicht - und diese Fälle liegen, was das subjektive Wohlbefinden angeht, oft weit auseinander. Insofern das moralisch Relevante an Kompensationsleistungen ist, dass jemand zusätzliche Belastungen auf sich nimmt, weil jemand anderes etwas tut, das er nicht hätte tun dürfen, kompensiert der moralische Vegetarier (dem Fleisch weiterhin gut schmeckt) das Fehlverhalten der Schlachter.

Die Struktur des Problems ist also dieses: Universalisierungsgebote verteilen die Lasten, die notwendig sind, um einen idealen Zustand zu erreichen, auf eine gewisse Weise - jeder muss so viel tun, dass der ideale Zustand erreicht würde, wenn jeder andere das Gleiche leistete. Kollektive Kompensationspflichten entstehen, falls solche Regeln befolgt werden müssen, deren nicht-universelle, aber zahlreiche Befolgung schon beste Konsequenzen hat tendenziell muss dann jeder der Befolger mehr leisten. Es ist leicht zu sehen, dass man Universalisierungsgebote akzeptieren kann, ohne kollektive Kompensationspflichten zu akzeptieren.

Aus der Sicht des Verallgemeinerbarkeitsgedanken scheint es mir naheliegend, das Befolgen von Regeln, deren vielfache Befolgung schon ideale Konsequenzen hat, als supererogatives Handeln zu verstehen: Wir alle haben die Pflicht, das zu tun, was bei gleicher Lastenverteilung jeder tun müsste, um das kollektiv beste Ergebnis zu erzielen. Wer mehr tut, geht (ceteris paribus ${ }^{12}$ ) über das moralisch Geforderte hinaus. Entsprechend: Wir alle haben den Unwert der Fleischproduktion vorausgesetzt - die Pflicht, keinem Tier aktiv zu Konsumzwecken zu schaden. Wer darüber hinaus auf den Konsum verzichtet, handelt supererogatorisch.

Die Idee der Verallgemeinerbarkeit moralisch erlaubter Handlungen kann also bestenfalls die Supererogativität einer vegetarischen Lebensweise etablieren. Handlungskonsequentialistische Überlegungen können, das haben wir im ersten Abschnitt gesehen, nicht einmal dies verlässlich tun: Wenn die Fleischproduktion hinreichend industriell betrieben wird, haben wir aus handlungskonsequentialistischer Perspektive sogar Grund, Fleisch von Großbetrieben statt von Kleinbetrieben zu kaufen. Mit diesen Überlegungen ist natürlich nicht endgültig gezeigt, dass sich eine moralische Pflicht, auf den

\footnotetext{
${ }^{12}$ Natürlich gibt es weiterhin echte Kompensationspflichten - wenn der Rettungsschwimmer passiv bleibt, muss der Urlauber das Kind retten. Hier geht es lediglich um Kompensationshandlungen, die hinsichtlich ihrer faktischen Konsequenzen keinen zusätzlichen Nutzen bringen.
} 
Konsum von Fleisch zu verzichten, nicht mit Bezug auf den Unwert der Produktionspraxis begründen lässt. Zumindest ist aber klar geworden, dass zwei sehr natürliche Kandidaten für die Etablierung einer engen normativen Beziehung zwischen der Praxis der Fleischproduktion und dem individuellen Konsumverhalten nicht das halten, was sie versprechen. Selbst wenn also der Unwert der Praxis der Fleischproduktion und der Kausalzusammenhang zwischen der Produktion und der Gesamtnachfrage zugestanden ist, wartet noch eine erhebliche moralphilosophische Herausforderung auf Vertreter der These, dass eine vegetarische Lebensweise moralisch geboten ist.

\section{Literatur}

Almassi, Ben (2011): „The Consequences of Individual Consumption: A Defence of Threshold Arguments for Vegetarianism and Consumer Ethics", in: Journal of Applied Philosophy 28,4: 396-411.

Devine, Philip (1978): „The moral basis of vegetarianism”, Philosophy 53: 481-505.

Glover, Jonathan (1975): „It Makes no Difference Whether or Not I Do It”, Proceedings of the Aristotelian Society, Supplementary Volumes, Vol. 49: 171-209.

Hooker, Brad (2000): Ideal Code, Real World. A rule-consequentialist Theory of Morality, Oxford: OUP.

Kagan, Shelly (2011): „Do I Make a Difference?”, Philosophy \& Public Affairs 39, 2: 105141.

Nefsky, Julia (2012): „Consequentialism and the Problem of Collective Harm: A Reply to Kagan”, Philosophy \& Public Affairs 39, 4: 364-395.

Regan, Tom (1980): „Utilitarianism, vegetarianism, and animal rights”, Philosophy and Public Affairs 9,4: 305-324

Schwartz, David T. (2010): Consuming Choices. Ethics in a Global Consumer Age. Plymouth: Rowman \& Littlefield.

Singer, Peter (1976): „Animal Liberation: A New Ethics for Our Treatment of Animals”. London: Jonathan Cape Ltd.

Singer, Marcus George (1961): Generalization in Ethics - An essay in the Logic of Ethics, with the Rudiments of a System of Moral Philosophy. New York: Alfred A. Knopf.

Wenz, Peter (1979): „Act utilitarianism and animal liberation”, The Personalist 60,4: $423-$ 428. 